\title{
A perspective for the interpretation of limbic system function
}

\author{
ROBERT L. ISAACSON \\ Department of Psychology, Center for Neurobehavioral Sciences, and The Clinical Campus \\ State University of New York at Binghamton, Binghamton, New York 13901
}

\begin{abstract}
The recent emphasis on neuromodulators may provide a new perspective for understanding the basis of action of the limbic system. A summary of neuromodulary effects on the hippocampus itself, as well as known hippocampal modulatory effects on the neuroendocrinic systems, is provided first. Then, evidence supporting the view that certain behavioral consequences of hippocampal damage can be alleviated by pharmacologic intervention which are also relatively slow in onset after damage is presented. Both are taken as evidence that destruction of the hippocampus produces some of its behavioral effects because of secondary reactions in other systems, probably components of the forebrain basal ganglia system. It is proposed that the normal function of the hippocampal formation may be to act as a neural neuromodulator of the basal ganglia and the hypothalamus.
\end{abstract}

The question of what brain mechanisms set mankind apart from other animals has not been resolved. Many have argued that it is the great development of the isocortex or the development of "association" areas within the isocortex. However, many animals have greater isocortical development than man, and some have higher ratios of association to sensorimotor cortex without seeming to be too intelligent. What may make man unique is the correlated development of both isocortical and limbic brain areas (see Douglas \& Marcellus, 1975). What interests me is why the enlargement of the limbic system, and especially the hippocampus, should confer such advanced capabilities when coupled with a large neocortical mass.

I would suggest that the key to such understanding is in the limbic system's ability to provide flexibility in neural systems by modulating them. It is now clear that many of the chemicals we used to call "neurotransmitters" do not act as fast-acting synaptic agents but as more slowly acting modifiers of activities in many thousands of cells in a more or less localized area. Thus, we have the notion of transmitters acting as "neuromodulators." In general, we tend to think of neuromodulators as agents like the biogenic amines and diffusely acting hormonal systems. However, it seems likely that certain neural systems may modulate synaptic activity in other brain regions. In other words, they might be called neural neuromodulators. In fact, all of the slowly acting neurotransmitter systems probably illustrate systems that are modulatory in nature. These would include monoamine and muscarinic cholinergic synapses in many systems.

In the past few years, the study of neuromodula-

Preparation of this paper was supported in part by Grant NSF BNS-781682 to Robert L. Isaacson. tory mechanisms has become a prominent direction of research in the neurophysiological areas. Many of the principles derived from such studies have farreaching significance. For example, in studies of the bullfrog sympathetic ganglion, slow changes in membrane conductance induced by one set of receptors can effect responsivity to activation of others, a heterosynaptic effect and one that is not cAMP dependent (Weight, Schulman, Smith, \& Busis, 1979). However, in Aplysia the modulatory action of the serotonergic metacerebral cell, which seems to exert widespread effects, does appear to involve this cyclic nucleotide (Kupferman, Cohen, Mandelbaum, Schonberg, Susswein, \& Weiss, 1979).

In addition, there is evidence that norepinephrine (NE) acts to modify GABA-induced inhibition in the cerebellum in a fashion that is independent of its own inhibitory effects (Woodward, Moises, Waterhouse, Hoffer, \& Freedman, 1979). In the neocortex of the rat, NE acts to enhance both GABA and acetylcholineinduced effects (Waterhouse, Moises, \& Woodward, 1978). The actions of NE seem to be both to enhance cellular discharges and to reduce the background levels of activity. Foote, Freedman, and Oliver (1975) were the first to describe this reduction in background activity to NE. The effect of this reduction would be to enhance the signal-to-noise ratio in decision theory terms. The action makes the detectability of changes easier because of the enhancement of the signal.

The NE input to the hippocampus seems to provide modulation of the responses of cells in the hippocampus that are dependent on rewarding events that may follow the signal (Segal \& Bloom, 1976). Another study by Segal (1976) has shown that ACTH can modify the responsiveness of hippocampal neu- 
rons to iontophoretically applied NE but not 5-HT This suggests a specific modulator mechanism for NE on these cells. The hippocampus seems to be a prime candidate for various modulating influences because of the convergence of adrenergic, serotonergic, and hormonal influences, especially ACTH and the corticosteroids.

There is evidence that neurally based neuromodulatory influences affect the hippocampus. For example, stimulation of the olfactory bulb only produces graduated potentials in the hippocampus, not unit discharges (Yokota, Reeves, \& MacLean, 1970). Graded potentials are also found after repetitive stimulation of other, well-established, afferent pathways to the hippocampus (Purpura, 1967; Spencer $\&$ Kandel, 1968). The fact that such a variety of afferent activation produces graduated membrane changes indicates the reality of neural neuromodulation of the hippocampus itself. However, neuromodulatory influences need not be entirely postsynaptic in nature. The long-lasting potentiation of activity in hippocampal efferent systems described originally by Bliss and L $\varnothing \mathrm{mo}(1973)$ is a presynaptic phenomenon that probably resulted from augmented transmitter release (Andersen, Sundberg, Sveen, \& Wigström, 1977).

Some of the most dramatic examples of the effects of the limbic system regulating neurohormonal modulators were provided by Bush, Lovely, and Pagano (1973). In this study, the deficit in the acquisition of avoidance responding by animals with amygdaloid damage could be overcome by replacement therapy with exogenous ACTH. This indicates that the behavioral changes produced by the lesion are not ones that should be considered "hard wired" deficits but, rather, ones for which there can be compensation by other mechanisms. In a later study, Lovely (1975) found that the enhanced avoidance acquisition of a two-way active avoidance task after hippocampal damage (but not septal) was shown to be depencient on the integrity of the pituitary gland. Such studies indicate that certain of the behavioral effects of lesions in these limbic areas at least represent an interaction of the regions with neurohumoral agents. They emphasize the strong limbic system association with the pituitary-adrenal axis.

There have been controversial results about the effects of hippocampal lesions on the release of corticosteroids under conditions of stress. We should not expect the interaction of the limbic system with endocrine systems to be simple. The situation, in fact, may be quite complicated. Recently, I reanalyzed some data from a study that was published several years ago (Lanier, Van Hartesveldt, Weiss, \& Isaacson, 1975). As a part of this study, plasma corticosterone was measured fluorometrically after ether stress. Resting levels of corticosterone were similar in all groups, and animals with dorsal or ventral hippocampal lesions, near-total hippocampal lesions, and two types of control animals all showed stress-induced increases in corticosterone levels. However, the levels of the stress-induced responses were higher in the two partial-lesion groups than in the nearly complete hippocampal lesion group ( $p<.01$, MannWhitney $U$ test) or the combined control groups $(p<.01$, Mann-Whitney U test). There were no differences between the near-total hippocampal lesion group and the controls. These results could be interpreted to indicate the existence of separate mechanisms related to the control of pituitary secretion in the hippocampus which normally may act in a type of synergism such that interruption of both results in no detectable changes in stress responses. Accordingly, the damage to dorsal regions and the damage to ventral regions would, in a sense, offset each other. However, it is likely that the disturbances created by neartotal lesions are more subtle than we have been able to detect in our experimental procedures.

It has been known for some while that lesions of the hippocampus can induce behavioral changes resembling those found after systemic administration of certain ACTH fragments (see de Wied, 1974). These changes are subtler in nature and found only under "low" arousal and incomplete training conditions. For example, resistance to extinction is found both after bilateral hippocampal damage and after $\mathrm{ACTH}_{4-10}(1)$, a condition in which arousal is assumed to be lower than in acquisition. Other parallels between the behavioral effects of the large hippocampal lesions and the hormonal treatment can be found.

Animals with hippocampal damage also are resistant to distractions when engaged in any type of learned response, have trouble in changing previously learned responses, and have increased locomotion and rates of response in operant situations (see Isaacson, 1974). Some of these characteristics can be "corrected" or improved to normal levels by the use of neuroleptic agents. These include chlorpromazine and haloperidol. An example comes from the work of Fish (1976). She found that the excessively high rate of responding on the $\mathrm{DRL}_{20}$ operant schedule produced in animals with hippocampal damage could be greatly reduced by haloperidol, but the number of reinforcements obtained by the animals was not greatly increased. An inhibitor of NE synthesis (FLA-63) did not produce this effect. Neither did serotonin depletion by PCPA.

In other tasks, haloperidol reduces the rate of responding but not the correctness of performance of the lesioned animals. Fish also used a two-choice brightness discrimination easily mastered by the normal rat but never learned by animals with hippocampal damage. Here the lesioned animals have high 
response rates that are reduced by haloperidol, but the number of correct responses is not improved by the drug.

Two points about the behavioral effects of hippocampal destruction should be made:

First, it is the enhanced rate of responding found after hippocampal destruction that is reduced by drugs capable of blocking dopaminergic receptors. The enhanced locomotion that usually follows bilateral hippocampal destruction is also reduced by the drugs. However, certain other changes in behavior produced by the lesion are not altered by neuroleptics.

Second, increased rates of responding and enhanced locomotion are not seen instantly after the lesion. Lanier and Isaacson (1975) tested animals with bilateral hippocampal destruction at different postoperative times. Enhanced locomotion in an open field peaks at about 8 days after the lesion and declines afterwards. The degree of reduction depends on the postoperative testing schedule. Campbell, Ballantine, and Lynch (1971) found that exaggerated responsiveness to amphetamine gradually developed after hippocampal lesions, reaching a maximum about 10 days after surgery. The subregion of the hippocampus that contributes most to the exaggerated responsiveness is the ventral hippocampus (Lanier \& Isaacson, 1975). It is from the more ventral areas of the hippocampus and subiculum that fibers reaching the hypothalamus and the nucleus accumbens take origin (Swanson \& Cowan, 1975, 1977).

The conclusion that must be drawn from a variety of data is that the hyperresponsiveness and enhanced levels of responding found after lesions of the hippocampus arise as a consequence of secondary changes induced in other regions of the central nervous system. Furthermore, these changes are not absolute but depend on the genetic constitution of the animal, its history, dietary base, and training-testing conditions.

\section{Areas Modulated by the Hippocampus}

As mentioned above, it seems likely that modulating influences exerted by the hippocampus are directed toward the mesolimbic-accumbens component of the ascending dopaminergic systems. The projections of the hippocampal formation, broadly defined so as to include subiculum, to nucleus accumbens now have been well documented in the rat (Ban \& Zyo, cited in Paletti \& Creswell, 1977; Raisman, 1966; Swanson \& Cowan, 1975), as well as in other species. The importance of these connections was emphasized both by the results from my laboratory, in which presumed dopaminergic antagonists alleviated some of the usual behavioral consequences of hippocampal destruction, and also because of similarities of effects of some types of dopaminergic stimulation to those found after large bilateral hippocampal lesions. These include the enhancement of ex- ploratory behavior after dopamine application to the nucleus accumbens (Jackson, Anden, \& Dahlstrom, 1975) and the enhancement of locomotion by the systemic administration of ET-495, a partial agonist of the dopamine system with predominant projections to the accumbens area (Cools \& Van Rossum, 1976). Other types of evidence could also be cited, including an enhanced responsiveness to d-amphetamine found about hippocampal lesions in certain behavioral situations (Woodruff \& Isaacson, 1977).

Approaching the matter from another point of view, Stevens has stressed the importance of influences on the striated complex (e.g., Stevens, 1979). She believes that schizophrenia is a disease that results in enhanced activity in the accumbens systems. The changes in accumbens could be due to alterations in the input from a number of limbic regions. Data supporting such a view include increased numbers of DA receptors in nucleus accumbens but not the caudate, as well as electrophysiological studies indicating abnormal activities along the mesolimbic-accumbens pathway in schizophrenic patients.

From the work on the interaction of the hippocampus with the pituitary adrenal access, it would also seem likely that important influences must reach the neurosecretory nuclei as well as the more posterior portions of the hypothalamus. Destruction of the hippocampus should lead to alterations in functional activities in these regions. However, these changes may be difficult to discover, since the interconnections will be largely modulatory and observable only under special conditions. Modulatory influences are difficult to detect in principle because they are interactive with other synaptic and nonsynaptic influences. The effects may be reflected in changes in background levels, on the release of transmitters, or on receptor receptivity and the reaction of postjunctional membranes. The influences could be an afferent terminals, interneurons, or major efferent cells. However, altered neuronal activities could be reflected in altered metabolic activities in the deprived area. In a similar fashion, there may be changes in regional cerebral blood flow or in membrane dynamics. There is no single way to determine these secondary changes in regions connected to a damaged area. The determination of secondary changes will depend on the convergence of information from a variety of approaches.

Recently, we have undertaken some studies to begin to understand the assumed secondary changes after hippocampal damage (Reinstein, Isaacson, \& Dunn, 1979). We began with attempts to understand the metabolic changes in different brain regions after bilateral hippocampal lesions, using the uptake of ${ }^{3} \mathrm{H}$-2-deoxy-D-glucose as the indicator of metabolic activity. Because of the alterations in response to stress and to environmental uncertainties found in animals with bilateral hippocampal lesions, we felt 
that we had to modify standard procedures, which require venous cannulation and the repeated handling of awake, unrestrained animals. Instead, we used techniques which we felt would adequately control the possibility of different amounts of the labeled 2DG reaching the brain.

To minimize the stress received by the animals, we used a subcutaneous injection of ${ }^{3} \mathrm{H}$-2-deoxy-D-glucose $\left({ }^{3} \mathrm{H}-2 \mathrm{DG}\right)$ in order to eliminate the need to implant chronic cannulae and to inject the ${ }^{3} \mathrm{H}-2 \mathrm{DG}$ intravenously. We also were concerned about restricting the movement of the animals or placing them in novel environments. The lesioned animals could respond in unusual manners to these stressful conditions. This would have confounded the basic lesioninduced secondary changes with altered stress reactions.

Three groups of male Long-Evans hooded rats were studied: Animals with bilateral hippocampal damage, animals with damage restricted to the neocortical surface overlying the hippocampus, and animals with sham operations. They were individually housed postoperatively for $\mathbf{3 0}$ days with food and water as desired. They were sacrificed $30 \mathrm{~min}$ after the subcutaneous injection of $20 \mu \mathrm{Ci}$ of ${ }^{3} \mathrm{H}-2 \mathrm{DG}$ on the 31 st day after surgery.

Eleven forebrain regions were analyzed. These were the olfactory bulb (OLF), the olfactory tubercule (OC), the frontal pole (FRO), the parietal cortex (PAR), the occipital cortex (OCC), the entorhinal cortex (ENT), the cingulate cortex (CIN), the striatum (CAU), the septal area (SEP), remaining portions of the most ventral hippocampus (HPC), and the hypothalamus (HYP). The 11 forebrain areas were separated over ice from the remainder of the forebrain after decapitation. The areas were weighed and placed into vials with Soluene-350 added. After some further processing, the tissue was evaluated for total disintegration per minute $(\mathrm{dpm})$ for each region. The dpms for each region were divided by the wet brain tissue weight and then normalized against the total dpm/weight for all of the brain sections studied for an individual animal. These normalized scores for each area for each group were then combined, and the results are presented in Figure 1.

Both animals with neocortical destruction and those with neocortical and hippocampal damage had reduced ${ }^{3} \mathrm{H}-2 \mathrm{DG}$ uptake in the occipital cortex. This was probably due to the interruption of normal blood supplies to the posterior neocortical surface by the lesion. Unique to the hippocampal lesion groups were reductions in uptake in the hypothalamus and a decrease in the olfactory bulb and tubercule. A change in hypothalamic uptake could have been anticipated because of the well-known relationship between the hippocampus and the hypothalamus, but the changes in the olfactory bulb were surprising. Recently, however, a direct projection from area $C_{A_{1}}$ of the hippocampus to the olfactory bulb and the anterior olfac-

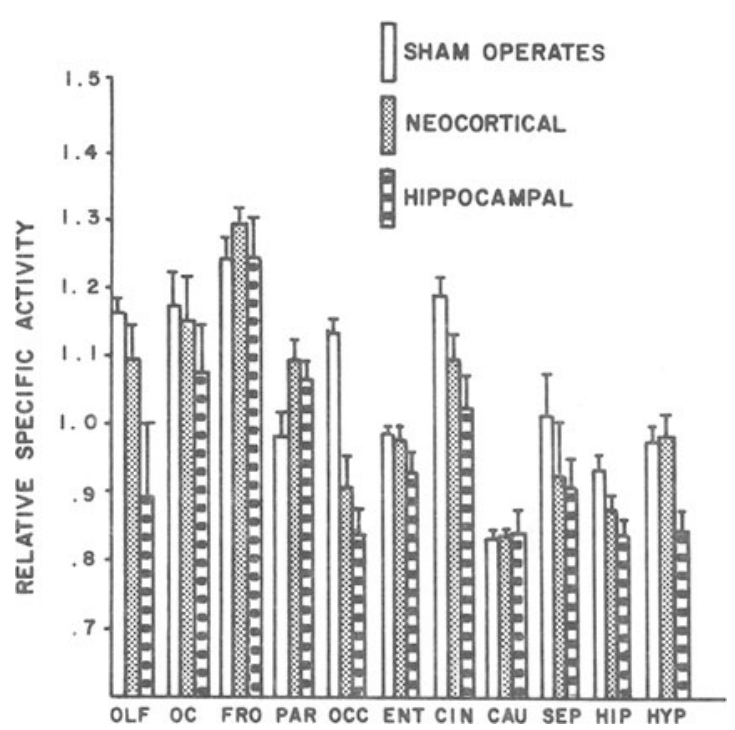

Figure 1. Relative uptake of $\left[{ }^{3} \mathrm{H}\right] 2$-deoxy-D-glucose in 15 sections of brains of rats with sham operations, neocortical lesions, or neocortical and hippocampal lesions. OLF, olfactory bulb; OC, olfactory cortex; FRO, frontal cortex; PAR, parietal cortex; OCC, occipital cortex; ENT, entorhinal cortex; CIN, cingulate cortex; CAU, caudate; SEP, septal area; HIP, hippocampus or remnants of hippocampus in lesioned animals; HYP, hypothalamus. (From Reinstein, Isaacson, \& Dunn, 1979.)

tory nucleus has been described (de Olmos, Hardy, \& Heimer, 1978). Delanoy and Dunn (1978) also found a decrease in the olfactory bulb after central administration of certain ACTH fragments. It is possible that the correspondence between certain of the effects of ACTH fragments and hippocampal damage may arise from both inducing alterations in the olfactory regions and further information collected.

There are many questions that need to be answered, and further information needs to be collected. In this initial study, $n$. accumbens was not isolated for evaluation. The striatal fragment that contained the caudate and globus pallidus did not show any selective effects. Since there are few interconnections between hippocampus and the caudate, we did not expect any change.

In the near future, we will be undertaking new studies with this method, considering additional brain regions and using different postoperative times, but we also realize that the labeled 2DG procedure will not give us all the information required to evaluate the neuromodulation theory. We plan to couple this method with other biochemical, electrical, and behavioral procedures.

\section{Modulation Contrasted with "Hard Wiring"}

It may be useful to discuss differences between a modulation approach to the study of lesions with more traditional views. In the history of the study of brain lesions related to behavior, the major search 
has been for regions that are essential parts of some behavioral systems. These efforts have included the search for motor and sensory regions, areas related to motivation and emotion, learning and memory, and even the special capability of using "spatial information." In all such cases, the hope has been to define what limited behavioral characteristics are permanently lost after a lesion, because if such a result were found, it would lead to attributing a special or unique characteristic to the neural area. Most investigators realize that such behavioral results would be suggestive at best and are aware that the procedure would be like trying to determine a function of a component in a computer or telephone switchboard by removing it.

Nevertheless, the search for structural functions continues with high intensity, and people ask, "What does region X really do?" To ask such a question presupposes that a reasonable answer could be made. The answer that would be satisfying to the asker would have to involve a machine with a set of parts with special operational characteristics. These parts would be presumed to be quite similar, if not identical, in all animals of a species and perhaps among a number of species. "Hard wire" models may allow for some relatively minor alterations resulting from developmental influences, but they are based on the view that, after some early period in life, the connections among neural components are fixed and permanent. If it were not this way, then the search for function by either lesion or stimulation techniques would be futile.

A modulation approach does not rule out the existence of some relatively specific input-output systems. Indeed, any reasonable theory of brain function must include some reasonably consistent sensory and motor pathways, even though they must be other than a direct periphery to central or central to peripheral connection. The considerable sensory processing that occurs along the multisynaptic afferent pathways and organization of motor movements in the brainstem and cord must be recognized. Even with these considerations, however, the fact remains that the central nervous system is largely a collection of interneurons, probably best considered as local circuit neurons, rather than cells involved with traditional sensory or motor systems. A modulation approach to limbic system function stresses the fact that biases would be established in cells of other grain systems by the limbic system but that they would not control or "drive" these other regions in a one-to-one manner. They would bias cells in these other regions to be more or less responsive to input arriving over other pathways or routes. This could be done by presynaptic, postsynaptic, metabolic, ionic, or other mechanisms, but the end result would be similar: A particular region becomes more or less ready to process or react to incoming information.
The effects of stimulation or lesions of a brain area can be interpreted as modifying the responsiveness of other areas, rather than as the removal of a component from an electronic circuit. The behavioral effects would be predictable only with the knowledge of the basis of ongoing states of activity in the structure, the nature of current afferent input, and ongoing motor activities, other modulating influences, and the like. Therefore, interference with a modulating system could not be counted on to produce uniformly consistent effects. They would depend on the factors influenced above, and, therefore, constitutional, historical, and testing factors would be critical determinants of the behavioral consequences of the interference. Empirical observations indicate this to be the case.

In addition, a modulatory perspective places the question of what a particular brain area does in a different light. The answer is that certain modulating influences are removed from other brain regions. The significance of this removal depends on a number of factors and can reveal itself in a number of different ways. What any limbic area does is bias and regulate, rather than control, direct, or even "store." The perspective is also in accord with Mountcastle's (1978) view that "it is predictable that, for higher nervous functions, one can localize a lesion but not a function"' (p. 26).

\section{REFERENCES}

Anderson, P., Sundberg, S. H., Sveen, O., \& Wigström, H. Specific long-lasting potentiation of synaptic transmission in hippocampal slices. Nature, 1977, 266, 736-737.

BLISS, T., \& LøMO, T. Long-lasting potentiation of synaptic transmission on the dentate area of the anesthetized rabbit following stimulation of the perforant path. Journal of Physiology, 1973, 232, 331-356.

Bush, D. F., Lovely, R. H., \& Pagano, R. R. Injection of ACTH induces recovery from shuttle-box avoidance deficits in rats with amygdaloid lesions. Journal of Comparative and Physiological Psychology, 1973, 83, 168-172.

Campbell, B., Ballantine, P., II, \& Lynch, G. Hippocampal duration of lesion effects and possible interactions with recovery after frontal cortical damage. Experimental Neurology, 1971, 33, 159-170.

Cools, A. R., \& VAN Rossum, J. M. Excitation-mediating and inhibition-mediating dopamine receptors: A new concept towards a better understanding of electrophysiological, biochemical, pharmacological, functional, and clinical data. Psychopharmacologia, 1976, 45, 243-254.

Delanoy, R. L., \& Dunn, A. J. Brain deoxyglucose uptake after footshock, ACTH analogues, $\alpha-\mathrm{MSH}$, corticosterone or LVP. Pharmacology, Biochemistry and Behavior, 1978, 9, 21-26.

de Olmos, J., Hardy, H., \& Heimer, L. The afferent connections of the main and the accessory olfactory bulb formations in the rat: An experimental HRP study. Journal of Comparative Neurology, 1978, 181, 213-244.

DE WIED, D. Pituitary-adrenal system hormones and behavior. In F. O. Schmitt \& F. G. Warden (Eds.), The neurosciences. Third study program. Cambridge: M.I.T. Press, 1974.

Douglas, R. J., \& Marcellus, D. The ascent of man: Deductions based on a multivariate analysis of brain. Brain, Behavior, 
and Evolution, 1975, 11, 179-213.

Fish, B. S. Catecholamine modulation of behavior following bilateral hippocampal damage. Unpublished $\mathrm{PhD}$ dissertation, University of Florida, 1976.

Foote, S. L., Freedman, R., \& Oliver, A. P. Effects of putative transmitters on neuronal activity in monkey cortex. Brain Research, 1975, 86, 229-242.

IsaAcson, R. L. The limbic system. New York: Plenum, 1974.

Jackson, D. M., Anden, N.-E., \& Dahlstrom, A. A functional effect of dopamine in nucleus accumbens and in some other dopamine-rich parts of the rat brain. Psychopharmacologia, 1975, 45, 151-155.

Kupferman, I., Cohen, J. L., Mandelbaum, D. E., Schonberg, M., Susswein, A. J., \& Weiss, K. R. Functional role of serotonergic neuromodulation in Aplysia. Federation Proceedings, 1979, 38, 2095-2102.

Lanier, L. P., \& IsaAcson, R. L. Activity changes related to the location of lesions in the hippocampus. Behavioral Biology, $1975,13,59-69$.

Lanier, L. P., Van Hartesveldt, C., Weiss, B. J., \& Isaacson, R. L. Effects of differential hippocampal damage of rhythmic and stress-induced corticosterone secretion in the rat. Neuroendocrinology, 1975, 18, 154-160.

Lovely, R. H. Hormonal dissociation of limbic lesion effects on shuttle avoidance in rats. Journal of Comparative and Physiological Psychology, 1975, 89, 224-230.

Mountcastle, V. B. Brain mechanisms for directed attention. Journal of the Royal Society of Medicine, 1978, 71, 14-28.

Paletti, C. E., \& Creswell, G. Fornix system efferent projections in the squirrel monkey: An experimental degeneration study. Journal of Comparative Neurology, 1977, 175, 101-127.

Purpura, D. P. Comparative study of dendrites. In G. C. Quarton, T. Melnechuck, \& F. O. Schmitt (Eds.), The neurosciences. A study program. New York: Rockefeller, 1967.

Raisman, G. The connections of the septum. Brain, 1966, 89, 317-348.

Reinstein, D. K., Isaacson, R. L., \& Dunn, A. J. Changes in 2-deoxy-D-glucose incorporation after neocortical and hippocampal destruction. Brain Research, 1979, 175, 392-397.

SEgAL, M. Interactions of ACTH and norepinephrine on the activity of rat hippocampal cells. Neuropharmacology, 1976, 15, 329-333.

Segal, M., \& Bloom, F. E. The action of norepinephrine in the rat hippocampus. IV. The effects of locus coeruleus stimulation on evoked hippocampal unit activity. Brain Research, 1976, 107, 513-525.

Spencer, W. A., \& Kandel, E. R. Cellular and integrative properties of hippocampal pyramidal cells and the comparative electrophysiology of cortical neurons. International Journal of Neurology, 1968, 3-4, 267-296.

Stevens, J. R. Schizophrenia and dopamine regulation in the mesolimbic systems. Trends in Neuroscience, 1979, April, pp. 102-105.

Swanson, L. W., \& Cowan, W. M. Hippocampal hypothalamic connections: Origin in subicular cortex, not Ammon's horn. Science, 1975, 189, 303-304.

Swanson, L. W., \& Cowan, W. M. An autoradiographic study of the organization of the efferent connections of the hippocampal formation in the rat. Journal of Comparative Neurology, 1977, 172, 44-84.

Waterhouse, B. D., Moises, H. C., \& Woodward, D. J. Noradrenergic modulation of somatosensory cortical neuronal responses to iontophoretically applied putative transmitters. Society for Neurosciences Abstracts, 1978, 4, 286.

Weight, F. F., Schulman, J. A., Smith, P. A., \& Busis, N. A. Long-lasting synaptic potentials and the modulation of synaptic transmission. Federation Proceedings, 1979, 38 , 2084-2094.

Woodruff, M. L., \& Isaacson, R. L. Attenuation of the ratedecreasing effects of amphetamine on fixed ratio responding in rats by hippocampal lesions. Behavioral Biology, 1977, 20, 493-499.

Woodward, D. J., Moises, H. C., Waterhouse, B. D., Hoffer, B. J., \& FreEdman, R. Modulatory actions of norepinephrine in the central nervous system. Federation Proceedings, 1979. 38, 2109-2116.

Yokota, T., Reeves, A. G., \& Maclean, P. D. Differential effects of septal and olfactory volleys on intracellular responses of hippocampal neurons in awake sitting monkeys. Journal of Neurophysiology, 1970, 33, 96-107. 